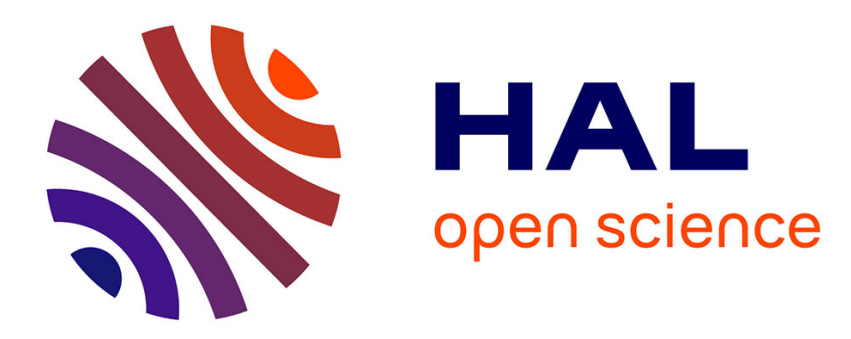

\title{
Hydrodynamics in Clay Nanopores
}

Alexandru Boţan, Benjamin Rotenberg, Virginie Marry, Pierre Turq, Benoît Noetinger

\section{To cite this version:}

Alexandru Boţan, Benjamin Rotenberg, Virginie Marry, Pierre Turq, Benoît Noetinger. Hydrodynamics in Clay Nanopores. Journal of Physical Chemistry C, 2011, 115 (32), pp.16109 - 16115. 10.1021/jp204772c . hal-01920439

\section{HAL Id: hal-01920439 https: / hal.sorbonne-universite.fr/hal-01920439}

Submitted on 13 Nov 2018

HAL is a multi-disciplinary open access archive for the deposit and dissemination of scientific research documents, whether they are published or not. The documents may come from teaching and research institutions in France or abroad, or from public or private research centers.
L'archive ouverte pluridisciplinaire $\mathbf{H A L}$, est destinée au dépôt et à la diffusion de documents scientifiques de niveau recherche, publiés ou non, émanant des établissements d'enseignement et de recherche français ou étrangers, des laboratoires publics ou privés. 


\title{
Hydrodynamics in clay nanopores
}

\author{
Alexandru Boţan, ${ }^{*, \dagger, \ddagger}$ Benjamin Rotenberg, ${ }^{*, \dagger}$ Virginie Marry, ${ }^{\dagger}$ Pierre Turq,${ }^{\dagger}$ and \\ Benoît Noetinger ${ }^{\ddagger}$ \\ Laboratoire PECSA, UMR 7195, 4 pl. Jussieu, Paris F-75005, France, and 1-4 Avenue de \\ Bois-Préau, Rueil-Malmaison 92852, France \\ E-mail: alexandru.botan@upmc.fr; benjamin.rotenberg@upmc.fr \\ Phone: +33 (0)144 273191. Fax: +33 (0)144 273228
}

\begin{abstract}
We report a molecular simulation study of hydrodynamics in clay nanopores, with pore widths ranging from 3 to $10 \mathrm{~nm}$. Understanding mass transfer through clay nanopores is necessary in many contexts such as groundwater hydrology, petroleum and gas reservoir engineering, as well as carbon dioxide sequestration or geological disposal of radioactive waste. Grand-canonical Monte-Carlo simulations first allow to determine the water content in the pores. We then analyze the structure and diffusion of confined water using equilibrium Molecular Dynamics. Finally, Non-Equilibrium MD allow to analyze the hydrodynamic behaviour of the confined fluid and assess the relevance of continuum hydrodynamics to describe the flow under a pressure gradient. The Navier-Stokes equation, using the density and viscosity of the bulk fluid, provides a reasonable description of the flow provided that the pore width is larger than $4 \mathrm{~nm}$ and that a slip boundary condition is used. We determine a slip length of $2.1 \AA$ at the clay surface. Although this value is small, neglecting slip in these nanopores results in large errors on the hydrodynamic flow. In the vicinity of the surface, the deviations from the

\footnotetext{
${ }^{*}$ To whom correspondence should be addressed

tUPMC-Paris6 and CNRS

$\ddagger$ IFP - Energies nouvelles
} 
prediction of the Navier-Stokes equation cannot be captured by a local viscosity determined from MD simulations.

\section{Introduction}

The behavior of fluids confined in nanopores is of great interest both from the fundamental and the technological point of view. Surface effects play a central role in processes such as wetting, impregnation and drying in interface and colloid science. Understanding mass transfer through clay pores is necessary in many contexts, such as groundwater hydrology, petroleum and gas reservoir engineering, as well as carbon dioxide sequestration or geological disposal of radioactive waste. Clays present a multiscale porosity, from the nanometer to the micrometer scale. Transport on large scales is controlled by the morphology of the material and the transport through the smallest pores. The upscaling from the pore-scale flow, usually described by the Navier-Stokes equation, to the macroscopic one, satisfying Darcy's law, can be obtained using the mathematical tools of homogenization. ${ }^{1-3}$

However, the relevance of a continuous description of hydrodynamics in nanopores must be questioned. It is indeed well known that confining a fluid into pores not much larger than the molecular size may lead to dramatic changes in its phase diagram, structure and dynamics. Interactions of the surface with the fluid may shift the liquid-vapour coexistence. ${ }^{4}$ The molecular nature of a liquid results in a layered structure in the vicinity of any surface. ${ }^{5}$ Finally, diffusion and hydrodynamic flow are influenced by the interaction with the surface. ${ }^{6}$ Molecular simulation is the method of choice to describe a fluid confined in nanopores. For example, Grand canonical Monte-Carlo and molecular dynamics simulations have allowed to predict the adsorption isotherms of water in clay interlayers or the structure and diffusion of water in clay interlayer (see e.g. ${ }^{7-9}$ for reviews) and in small interparticle pores. ${ }^{10-15}$

Non-equilibrium molecular dynamics (NEMD) simulation has allowed to investigate the validity of continuous hydrodynamics of simple systems under confinement. ${ }^{6}$ Travis et al. simulated a 
Poiseuille flow of a Lennard-Jones fluid through slit pores with two different widths ${ }^{16}$ and demonstrated that for channels smaller than $10 \mathrm{LJ}$ diameters the velocity profiles are not well described by the Navier-Stokes prediction. Similar findings where reported by Di Leo and Marañón ${ }^{17}$ for water in a 3-nm slit pore (about 10 times the size of a water molecule). Molecular simulations have also been used to address another crucial aspect of hydrodynamics, namely the choice of boundary condition at the solid/liquid interface. Since the pioneering work of Bocquet and Barrat on the derivation of a slip length from molecular simulations, ${ }^{18-20}$ numerous work investigated its dependence on the solid roughness or on wall/fluid and fluid/fluid interactions. Sendner et al. reported the variation of the slip length for water in a model slit pore as a function of the wettability, by tuning the fluid/wall interaction and thereby changing the contact angle. ${ }^{21}$ They found that a stick boundary condition could be used only for weak fluid/wall interactions. Huang et al. simulated Couette flows to investigate the dependence of the slip length on the surface charge density of a model surface ${ }^{22}$ and found that the slip length decreassd with increasing surface charge density. Voronov et al. reviewed studies of the effects of roughness on the slip length, and concluded that roughness can lead to both an increase or decrease slip, all other things being equal. ${ }^{23}$ In all the above studies, the effect of the surfaces on the density of the confined fluid was not considered prior to the investigation of the hydrodynamic behaviour.

We report here a systematic molecular simulation study of water adsorption and transport in interparticle pores of sodium montmorillonite clays, with sizes ranging from 3 to $10 \mathrm{~nm}$. For each pore size, we first determine the water density from Monte-Carlo simulations in the $\mu_{\mathrm{H}_{2} \mathrm{O}} V T$ ensemble. We then calculate the density profile and the radial distribution functions, as well as diffusion properties, via equilibrium molecular dynamics. Finally, we investigate using non-equilibrium molecular dynamics the hydrodynamics of water confined in clay nanopores. More precisely, we address the following questions : How far from the surface is the fluid velocity profile well described by the continuous prediction (e.g. Poiseuille flow)? Is the use of the bulk density and viscosity of the fluid appropriate to determine the properties of the confined fluid? Can the continuous description be improved using locally varying properties (density, viscosity) and/or boundary 
conditions (slip length) determined from molecular simulations?

\section{Models and Methods}

In the present calculations, we study the sodium-saturated Wyoming montmorillonite with unit formula

$$
\mathrm{Na}_{0.75}^{+}\left[\mathrm{Si}_{8}\right]\left(\mathrm{Al}_{3.25} \mathrm{Mg}_{0.75}\right) \mathrm{O}_{20}(\mathrm{OH})_{4},
$$

the atomic coordinates and force field are determined by Skipper ${ }^{24}$ and Smith. ${ }^{25}$ The single clay layer used in simulations consists of $8 \times 4$ unit cells (totalling 1280 atoms) with a thickness 6.54 $\AA$ that corresponds $41.44 \times 35.88 \AA^{2}$ in the $x-y$ plane. The clay layer is divided in two halflayers located at the top and bottom of the box, and forms a section of slit-like pore. The box is periodically replicated in all directions. The space between the half-layers contains water and $\mathrm{Na}^{+}$, as shown in Figure 1. Water is described by the extended simple point charge model SPC/E. ${ }^{26} \mathrm{We}$ perform the simulations for different pore widths: $3 \mathrm{~nm}, 4.5 \mathrm{~nm}, 6 \mathrm{~nm}, 8 \mathrm{~nm}$ and $10 \mathrm{~nm}$, where the width is defined as the distance between the $\mathrm{Al}$ atoms in each half-layer.

All interactions between particles are described by Lennard-Jones (LJ) and Coulomb potentials:

$$
U\left(r_{i j}\right)=4 \varepsilon_{i j}\left[\left(\frac{\sigma_{i j}}{r_{i j}}\right)^{12}-\left(\frac{\sigma_{i j}}{r_{i j}}\right)^{6}\right]+\frac{q_{i} q_{j}}{4 \pi \varepsilon_{0} r_{i j}},
$$

where $r_{i j}$ is the distance between sites $i$ and $j$ of different molecules, $q_{i}$ is the partial charge of the site, $\sigma_{i j}$ and $\varepsilon_{i j}$ are LJ parameters deduced from the conventional Lorentz-Berthelot mixing rules. ${ }^{27}$ We use a cut-off radius of $10 \AA$ for LJ interactions and Ewald summation to compute long-range electrostatic interactions.

\section{Monte-Carlo simulations}

The amount of water adsorbed for each pore width is determined from grand canonical MonteCarlo (GCMC) simulations using configurational bias techniques. ${ }^{27,28}$ Details of the GCMC al- 
gorithm and configurational bias can be found in. ${ }^{29}$ In the present study, the pore water is in contact with a reservoir which sets its chemical potential. The temperature and pressure of the water in reservoir are fixed at $300 \mathrm{~K}$ and $1 \mathrm{~atm}$, corresponding to a bulk density of $0.997 \mathrm{~g} / \mathrm{cm}^{3} .30$ The value of the chemical potential was first estimated from GCMC simulation of water bulk phase at $T=300 \mathrm{~K}$ in a cubic box of size $L=40 \AA$. (resulting in $\approx 2000 \mathrm{H}_{2} \mathrm{O}$ molecules). For $\mu_{H_{2} O}=49.0 \mathrm{~kJ} / \mathrm{mol}$ the obtained density $\left(\rho=0.994 \pm 0.006 \mathrm{~g} / \mathrm{cm}^{3}\right)$ agrees with the experimental one within the error bar and we thus use this chemical potential for the subsequent GCMC simulations in clays. For the latter, the system is equilibrated for $10^{8}$ steps and $5 \times 10^{7}$ additional steps are used to compute the composition.

\section{Equilibrium Molecular Dynamics}

Once the water content is known, we use molecular dynamics (MD) ensemble to study the structure and diffusion of the confined fluid. All MD simulations (including non-equilibrium dynamics) are carried out with the LAMMPS ${ }^{31}$ simulation package in the canonical ensemble. Initial configurations are taken from Monte Carlo simulations. A constant temperature of $300 \mathrm{~K}$ is maintained with a Nose-Hoover ${ }^{27}$ thermostat and the equations of motion for the rigid SPC/E are integrated using the SHAKE algorithm. ${ }^{32}$ The trajectories of atoms for $\mathrm{H}_{2} \mathrm{O}$ and $\mathrm{Na}^{+}$are collected every $0.1 \mathrm{ps}$ for $4.5 \mathrm{~ns}$, after an equilibration period of $2 \mathrm{~ns}$.

For the radial distribution functions of O-atoms, we divide the pore volume into layers, each of which is a slab parallel to the surface $(x-y$ plane) of thickness $0.5 \AA$. O-atoms in each layer contribute to the two-dimensional RDF of the layer:

$$
g(z, r)=\frac{n(r, r+\Delta r)}{\rho \Delta A}
$$

where $\Delta A=2 \pi r \Delta r$ is the area of a ring bounded by circles with $r$ and $r+\Delta r$ radii, $\rho$ is the twodimensional particle density. The value $n$ denotes the number of $\mathrm{O}$ atoms with a distance to the center atom in $[r: r+\Delta r]$. 
Self-diffusion coefficients are calculated according to the method introduced by Liu et al. ${ }^{33}$ Due to symmetry of the system, the diffusion tensor contains two components: parallel $\left(D_{\|}\right)$and perpendicular $\left(D_{\perp}\right)$ to the clay surface. Since the properties of the confined fluid depend on the distance from the surface, we divide the pore into layers, in which we compute $D_{\|}$and $D_{\perp}$. We calculate $D_{\|}$from the mean-square displacement of molecules in each layer, centered at a distance $z_{i}$ from the surface. To that end we introduce a function $S_{C}^{i}$ whose value is 1 if the molecule remains continuously in the $i$-th layer during the interval $[0 ; \mathrm{t}]$ and 0 otherwise. Then, we have:

$$
D_{\|}\left(z_{i}\right)=\lim _{t \rightarrow \infty} \frac{\left\langle\left[\Delta x^{2}(t)+\Delta y^{2}(t)\right] S_{C}^{i}(t)\right\rangle}{4 t\left\langle S_{C}^{i}(t)\right\rangle} .
$$

In the direction perpendicular to the surface, the equation for diffusion coefficient is expressed through the correlation:

$$
D_{\perp}\left(z_{i}\right)=-\left(\frac{L}{n \pi}\right)^{2} \lim _{t \rightarrow 0} \frac{1}{t} \ln \frac{\left\langle\Psi_{n}^{i}(t) \Psi_{n}^{i}(0) S_{C}^{i}(t)\right\rangle}{\left\langle S_{C}^{i}(0)\right\rangle}
$$

with $\Psi_{n}^{i}(t)=\sin \left(n \pi\left(z(t)-z_{\text {min }}^{i}\right) / L\right)$, where $n$ is an integer, $L=z_{\text {max }}^{i}-z_{\text {min }}^{i}$ and $z_{\text {max }}^{i}$ and $z_{\text {min }}^{i}$ are the boundaries of the $i$-th layer. Further details of the diffusion calculation are described elsewhere. ${ }^{10,34}$

\section{Non-Equilibrium Molecular Dynamics}

Finally, we use non-equilibrium molecular dynamics to investigate the hydrodynamic properties of the confined fluid. More precisely, we want to assess the relevance of a continuous description to describe the hydrodynamic flow arising from a uniform pressure gradient. The usual ansatz in molecular simulation consists in applying a constant external force, parallel to the surface, to each fluid atom. We use a force $F_{x}=0.7510^{-3} \mathrm{kcal} / \mathrm{mol} / \AA$, in the range corresponding to a linear response of the system (not shown). There are two additional differences compared to the equilibrium simulations. On the one hand, the thermostat is coupled only to the degree of freedom of the fluid in the $y$-direction, perpendicular to the force. On the other hand, periodic boundary 
conditions are applied only in the $x$ and $y$ directions. To that end we complete the two clay layers as indicated by the shaded atoms in Figure 1, to ensure that the thickness of the layers exceeds the cut-off radius $R_{c}=10 \AA$. Steady state is reached in a time that depends on the pore size and does not exceed 2 ns. The velocities of the particles are then sampled for 6 ns in bins of width $0.5 \AA$.

The shear viscosity is estimated by two approaches. ${ }^{35}$ The first one assumes a constant density and viscosity in the pore, a valid approximation sufficiently far from the surface. The steadystate Stokes equation predicts a parabolic (Poiseuille) velocity profile, whose curvature allows to determine the viscosity:

$$
\eta=-\frac{F_{x} \rho_{V}}{\left(\frac{\mathrm{d}^{2} v_{x}}{\mathrm{~d} z^{2}}\right)}
$$

where $\rho_{V}$ is the number density. The second approach uses the definition of viscosity from the local shear rate $\gamma(z)=\frac{\mathrm{d} v_{x}}{\mathrm{~d} z}$ and the pressure tensor $P_{x z}(z)=F_{x} \int_{0}^{z} \rho_{V}\left(z^{\prime}\right) \mathrm{d} z^{\prime}$ :

$$
\eta(z)=-\lim _{F_{x} \rightarrow 0} \frac{P_{x z}(z)}{\gamma(z)}
$$

\section{Results and Discussion}

\section{Static properties}

Figure 2 reports the number density profiles of water and ions as well as the mass density profile of the fluid, for a pore width $H=4.5 \mathrm{~nm}$. Density oscillations near the surface for other pore widths are identical (not shown). The z-axis on this figure is limited from two sides by the location of the clay surface oxygens. The maxima of the mass density at 2.7, 4.3, 6.0 and $9.3 \AA$ from the surface, coincide with that of the number density of water oxygen, except for the second one corresponding to the $\mathrm{Na}^{+}$ions.

The comparison of water density profile among different types of surface of slit-like pore such as rutile, ${ }^{36}$ laponite, ${ }^{14}$ muscovite mica, ${ }^{11}$ carbon $^{37}$ demonstrates that the nature of the surface modulates the position, width and intensity of the peaks. However, the distance from the surface 
over which the density oscillations decay, approximately equal to $12 \AA$ (independently of the pore width), is almost independent of the surface. Further away from the surface, the density reaches a constant value: $0.982 \mathrm{~g} / \mathrm{cm}^{3}(H=3 \mathrm{~nm}), 0.985 \mathrm{~g} / \mathrm{cm}^{3}(H=4.5 \mathrm{~nm})$ and $0.983 \mathrm{~g} / \mathrm{cm}^{3}$ for $H=6$, 8 and $10 \mathrm{~nm}$. Within the accuracy of estimates $(\approx 1 \%)$, we conclude that the pore size does not influence the density of the fluid in the center of the pore. These values are close to the bulk one for the same chemical potential and temperature $\left(0.994 \pm 0.004 \mathrm{~g} / \mathrm{cm}^{3}\right)$.

Figure 3 shows two-dimensional radial distribution functions (rdf) of O-atoms in layers parallel to the surface, computed from an 8-ns equilibrium MD simulation of the $10 \mathrm{~nm}$ pore. The inset of Figure 3 illustrates the location of the layers. The first 3 layers are centered at 2.7, 4.3 and $6.0 \AA$ from the surface, i.e. the positions of the first 3 density maxima. Three additional layers are located in the region of constant density at 13,24 and $33 \AA$ from the surface. As expected, deviations in the rdf from bulk value are only observed in the three surface layers and the fluid further away from the surface is identical to that in bulk water.

\section{Diffusion}

To investigate how diffusion is affected by the confinement, we computed the components of the diffusion tensor in layers defined as follows. The first three layers are bounded by [0:4.2] $\AA$, [4.2:7.8] $\AA$ and [7.8:13.7] $\AA$ from the plane of surface oxygen atoms, that correspond to locations of the maxima in the number density of water. The others are located in the regions with a constant density, as illustrated in Figure 4. $D_{\|}$and $D_{\perp}$ are then computed for each layer as described in section "Equilibrium Molecular Dynamics".

The results are given in Figure 4, which presents $D_{\|}$and $D_{\perp}$ as a function of the distance from the surface. The diffusivity close to the surface differs significantly from the values in the middle of the pore. This can be explained by the higher density of the fluid and H-bonds donated by water molecules to the surface oxygen atoms. ${ }^{10}$ However, $D_{\|}(\mathrm{z})$ reaches a minimum value only in the second layer, indicating that the hydration of $\mathrm{Na}^{+}$atoms has a large effect. In the absence of cations, the diffusivity monotonically increases from the surface to the center of the pore. ${ }^{36}$ 
In the fourth and next layers both components are almost constant and $D_{\|}>D_{\perp} \approx D_{b u l k}=2.80 \pm 0.0510^{-9} \mathrm{~m}^{2} \mathrm{~s}^{-}$ The bulk value reported here corresponds to a simulation of 2000 molecules at the same density as in the center pore center. Note that the higher value compared to that often reported for the SPC/E model is not due to the slightly smaller density compared to the experimental one, but to the sizedependence of the diffusion coefficient. ${ }^{38}$ Indeed, the latter are usually computed for small box sizes compared to the one simulated here. A study of the size-dependence for SPC/E by Kerisit et al. ${ }^{39}$ concluded to an extrapolated value of $2.8410^{-9} \mathrm{~m}^{2} \mathrm{~s}^{-1}$ for an infinite box size. The excess of $D_{\|}$over $D_{\perp}$ in the center of the pore, already observed with other systems ${ }^{36,40}$ is most likely an effect of the asymmetry of the simulation box, which is twice larger in the direction perpendicular to the surface than in the directions parallel to the surface.

\section{Shear viscosity}

We now turn to the hydrodynamic properties of water confined in clay nanopores and assess the relevance of continuous hydrodynamics on the nanoscale. In non-equilibrium Molecular Dynamics simulations, a constant force parallel to the surface is applied to each fluid atom, as described in section "Non-Equilibrium Molecular Dynamics", inducing steady-state velocity profiles reported in Figure 5 for pore widths $H=3,4.5,6,8$ and $10 \mathrm{~nm}$. Assuming a uniform density and viscosity of the fluid, the Navier-Stokes equation predicts a parabolic (Poiseuille) velocity profile. The simulated profiles are indeed parabolic in the center of the pore and the curvature provides the viscosity of the confined fluid, according to Eq. (1). The resulting values are: $\eta=0.70 \pm 0.07 \mathrm{cP}$ for $4.5 \mathrm{~nm}, 0.68 \pm 0.02 \mathrm{cP}$ for $6 \mathrm{~nm}, 0.709 \pm 0.005 \mathrm{cP}$ for $8 \mathrm{~nm}$ and $0.685 \pm 0.003 \mathrm{cP}$ for $10 \mathrm{~nm}$. In the $3 \mathrm{~nm}$ pore, the region of constant density is too small $(<3 \AA)$ to provide an estimate of the viscosity. For comparison, we also computed the viscosity of bulk water with the same density $\left(\rho_{m}=0.983 \mathrm{~g} / \mathrm{cm}^{3}\right.$ and $\left.\mathrm{T}=300 \mathrm{~K}\right)$ and under normal conditions $\left(\rho_{m}=0.997 \mathrm{~g} / \mathrm{cm}^{3}\right.$ and $\left.\mathrm{T}=300 \mathrm{~K}\right)$ from the Green-Kubo equation, ${ }^{41}$ with the result $0.66 \pm 0.02 \mathrm{cP}$ and $0.67 \pm 0.02 \mathrm{cP}$, correspondingly. Within the accuracy of these estimates, the viscosity of the confined fluid in the center of the pore is thus equal to that of the bulk fluid with the same density. 
However, deviations from a parabolic flow profile are observed in the vicinity of the surface. To analyze these deviations, we sampled more accurately the velocity profiles for two pore sizes, collecting the velocities for $40 \mathrm{~ns}$ instead of $6 \mathrm{~ns}$ in bins of width $0.1 \AA$ instead of $0.5 \AA$. The results for $H=4.5 \mathrm{~nm}$ are reported in Figure 6a. The velocity profile is parabolic only in the center of the pore and oscillates closer to the surface. The velocity maxima coincide with that in the density. Moreover, the larger the deviation from the bulk density, the larger the deviation from the parabolic velocity profile. Such oscillations arise from the discrete (molecular) nature of the fluid and have even been observed for a confined hard-sphere fluid. ${ }^{42}$

A possible improvement of the continuous description consists in computing the local viscosity of the fluid as the ratio between the shear stress and the shear rate (Eq. (2)). To estimate the latter, we first fit the simulated density profile to a piecewise polynomial, shown in Figure 6a. The shear rate is then the derivative of this polynomial, presented in Figure 6b, together with the pressure

tensor $P_{x z}(z)=F_{x} \int_{0}^{H / 2} \rho_{V}(z) d z$. Figure 6c shows the resulting viscosity profile. As expected, the viscosity is uniform in the middle of the pore and the value of $0.67 \mathrm{cP}$ agrees with both the first estimate from Eq. (1) and the one obtained in the bulk fluid $(0.66 \pm 0.02 \mathrm{cP})$. Closer to the surface the viscosity oscillates and even becomes negative. Such unphysical values indicate that the hydrodynamic behaviour of the fluid close to the surface cannot simply be described by a local viscosity defined by Eq. (2).

\section{Boundary condition}

Since the velocity profile is parabolic at a distance larger than $\approx 1 \mathrm{~nm}$ from the surface, two questions arise. Firstly, to which boundary condition do the simulation results in the center of the pore correspond? Secondly, do the deviations from the parabolic profile in the vicinity of the surface contribute significantly to the overall flow? The usual "stick" boundary condition corresponds to a vanishing fluid velocity at the solid/fluid interface. When this is not the case, a slip boundary 
condition can be introduced, characterized by the slip length :

$$
b= \pm \frac{v_{x}\left(z_{\text {surf }}\right)}{\left(\frac{\mathrm{d} v_{x}}{\mathrm{~d} z}\right)_{z_{\text {surf }}}}
$$

This requires the knowledge of the location $z_{\text {surf }}$ of the surface where the hydrodynamic boundary condition is applied. Its definition for atomistically resolved surfaces requires some caution. Several options can be considered.

(1) The first one was introduced by Bocquet and Barrat, ${ }^{43}$ who derived a Green-Kubo expression for the location of the fluid-solid interface :

$$
z_{\text {surf }}=\frac{\int_{0}^{\infty} d t\left\langle F_{\|}(t) P_{x z}(0)\right\rangle}{\int_{0}^{\infty} d t\left\langle F_{\|}(t) F_{\|}(0)\right\rangle},
$$

where $F_{\|}$is the tangential force exerted by the wall on the fluid. A similar expression was also derived for the associated slip length. The numerical convergence of these integrals remains however a challenge even for a simple Lennard-Jones fluid, for which $z_{\operatorname{surf}}$ was found to be of the order of one atomic layer inside the liquid. ${ }^{19,43}$

(2) A second point of view is to consider that $z_{\text {surf }}$ corresponds to a partitioning of space between a region where the fluid is present and homogeneous and another one without any liquid. This is the definition of the Gibbs dividing surface, according to :

$$
\int_{0}^{z_{\text {surf }}}\left[\rho_{\text {bulk }}-\rho(z)\right] \mathrm{d} z=\int_{z_{\text {surf }}}^{+\infty} \rho(z) \mathrm{d} z
$$

From the density profiles we find $z_{\text {surf }}$ at $1.2 \AA$ closer to the center of the pore than the surface oxygen atoms.

(3) Finally, an intuitive definition of $z_{\text {surf }}$ consists in splitting the pore into a region where the fluid behaves as in the bulk and another region where it behaves differently due to the proximity of the surfaces. The choice of $z_{\text {surf }}$ is then rather arbitrary. From the previous sections, a possible 
choice would be $z_{\text {surf }}=1 \mathrm{~nm}$, since this corresponds to a region where the structure and the dynamics differ significantly from the bulk. Because the "surface fluid" contained in this region is not immobile, such a model must account for a "surface flux", which can be determined from MD simulations in addition to $z_{\text {surf }}$ and $b$. In the $4.5 \mathrm{~nm}$ pore, this flux contributes approximately $40 \%$ of the total flux and $13 \%$ in the $8 \mathrm{~nm}$ pore. Moreover, it should be stressed that the slip length defined by Eq. (3) then depends on the size of the pore, rendering its interpretation as an intrinsic property of the wall/fluid interface rather difficult.

Considering the numerical challenge to quantify properly $z_{\text {surf }}$ and $b$ with the first option and the arbitrariness of the last one, we restrict ourselves here to identifying the surface where the hydrodynamic boundary condition applies with the Gibbs dividing surface defined by Eq. (5). This "physical" definition also corresponds directly to the continuous model underlying the use of the Navier-Stokes equation in its simplest form, with a homogeneous fluid characterized by its bulk density and viscosity. In particular, this assumes that the parabolic velocity profile in the bulk region extends even within the region where the fluid is known from our molecular simulations to be inhomogenous and not well described by a purely viscous behaviour (see Figure 6a and Figure 6c). Nevertheless, the parabolic fit to the simulated velocity profile near the center of the pore, already used to measure the viscosity in the previous section, describes reasonably well (see also below) the simulation results closer to the surface, as can be seen on Figure 7.

From this parabolic profile, the slip length is then determined via Eq. (3) by computing the velocity and its derivative at $z_{\text {surf }}$. Comparing the result for different pore widths, we obtain the following estimate of the slip length: $b=2.1 \pm 0.05 \AA$. A slip boundary condition in clays had already been introduced by Marry et al., ${ }^{44}$ who investigated electro-osmosis in Na-montmorillonite with the same force field and model of water from equilibrium molecular simulations. However, the reported value of the slip length was $6 \AA$. Several reasons can explain this discrepancy: a different value of $z_{\text {surf }}(2.4 \AA$ vs $1.2 \AA$ in this work), the determination of the Navier-Stokes profile using the experimental value of the viscosity ( $0.9 \mathrm{cP}$ vs $0.67 \mathrm{cP}$ for SPC/E water model) and the numerical issues related to the evalution of non-equilibrium quantities from equilibrium 
simulations using Green-Kubo relations. While there are no experimental determination of the slip length on montmorillonite clays, we can make a tentative comparison with experimental data using the connection proposed by Sender et al. for varying water/wall interactions between the slip length and the contact angle. ${ }^{21}$ The contact angle corresponding to our value of the slip length does not exceed $60^{\circ}$, in agreement with the experimental results of Lopez-Duran et al. ${ }^{45}$

While the slip length may seem small, neglecting slip could result in small pores in a large error on the overall flow. To address this issue we compare the total fluid flow obtained from MD simulations to the one predicted by the parabolic profiles, solutions of the Stokes equation with stick $\left(J_{\text {stick }}\right)$ and slip $\left(J_{\text {slip }}\right)$ boundary conditions. The corresponding velocity profiles are illustrated on Figure 7. The relative errors with both models and two pore sizes are summarized in Table 1. Neglecting slip results in errors in the overall flow, which is the quantity of interest for upscaling, that exceed $10 \%$ even for the largest pores. However, our results also demonstrate that it is possible to correctly describe the flow in pores of a few nm only using a Poiseuille flow with slip boundary conditions, with the slip length determined from molecular simulation.

\section{Conclusions}

We demonstrated using Grand-Canonical Monte-Carlo simulations that the water density in the center of clay nanopores $(3-10 \mathrm{~nm})$ is similar to that of bulk water. The width of the region perturbed by the surface extends over approximately $1.2 \mathrm{~nm}$. The Navier-Stokes equation, using the density and viscosity of the bulk fluid, provides a reasonable description of the hydrodynamic flow under an external force provided that the pore size is larger than $4 \mathrm{~nm}$ and that a slip boundary condition is used. We discussed the choice of the surface where the hydrodynamic boundary condition should be applied and determined a slip length of $\approx 2.1 \AA$ at the clay surface. Although this value is small, neglecting slip in these nanopores results in large errors on the overall flow. In the vicinity of the surface, the deviations from the prediction of the Navier-Stokes equation cannot be captured by a local viscosity determined from MD simulations. 


\section{Acknowledgement}

We thank Prof. J.-F. Dufrêche for valuable discussions. BR, VM and PT acknowledge financial support from the Agence Nationale de la Recherche under grant ANR-09-SYSC-012.

\section{References}

(1) Auriault, J.; Adler, P. Adv. Wat. Resour. 1995, 18, 217 - 226.

(2) Allaire, G. Arch. Rational Mech. Anal. 1991, 113, 261-298.

(3) Moyne, C.; Murad, M. A. Int. J. Solids Struct. 2002, 39, 6159-6190.

(4) Brovchenko, I.; Oleinikova, A. Interfacial and confined water; Elsevier: Amsterdam, 2008.

(5) Quirke, N. Adsorption and transport at the nanoscale; Taylor and Francis Group: New York, 2006.

(6) Karniadakis, G.; Beskok, A.; Aluru, N. Microflows and Nanoflows. Fundamentals and Simulation; Springer: New York, 2005.

(7) Auerbach, S. M.; Carrado, K. A.; Dutta, P. K. Handbook of Layered Materials, 1st ed.; M. Dekker: New York, 2004.

(8) Skipper, N. T.; Locka, P. A.; Titiloye, J. O.; Swenson, J.; Mirza, Z. A.; Howells, W. S.; Fernandez-Alonso, F. Chem. Geol. 2006, 230, 182-196.

(9) Smirnov, K. S.; Bougeard, D. J. Phys.: Condens. Matter 2010, 22, 284115.

(10) Marry, V.; Rotenberg, B.; Turq, P. Phys. Chem. Chem. Phys. 2008, 10, 4802-4813.

(11) Sakuma, H.; Kawamura, K. Geochim. Cosmochim. Acta 2009, 73, 4100-4110.

(12) Kalinichev, A.; Wang, J.; Kirkpatrick, R. J. Cement and Concrete Research 2007, 37, 337347. 
(13) Ichikawa, Y.; Kawamura, K.; Nakano, M.; Kitayama, K.; Kawamura, H. Engineering Geology 1999, 54, 21-31.

(14) Leote de Carvalho, R. J. F.; Skipper, N. T. J. Chem. Phys. 2001, 114, 3727.

(15) Choi, J. H.; Faisal Anwar, A. H. M.; Kawamura, K.; Ichikawa, Y. Int. J. Numer. Anal. Meth. Geomech. 2009, 33, 687-707.

(16) Travis, K. P.; Todd, B. D.; Evans, D. J. Phys. Rev. E 1997, 55, 4288-4295.

(17) Di Leo, J. M.; Marañón, J. Int. J. Quant. Chem. 2008, 108, 1623-1628.

(18) Bocquet, L.; Barrat, J.-L. Phys. Rev. Lett. 1993, 70, 2726-2729.

(19) Barrat, J.-L.; Bocquet, L. Faraday Discuss. 1999, 112, 119-128.

(20) Bocquet, L.; Barrat, J.-L. Soft Matter 2007, 3, 685-693.

(21) Sendner, C.; Horinek, D.; Bocquet, L.; Netz, R. R. Langmuir 2009, 25, 10768-10781.

(22) Huang, D. M.; Cottin-Bizonne, C.; Ybert, C.; Bocquet, L. Langmuir 2008, 24, 1442Ü-1450.

(23) Voronov, R. S.; Papavassiliou, D. V.; Lee, L. L. Ind. Eng. Chem. Res. 2008, 47, 2455-2477.

(24) Skipper, N. T.; Refson, K.; McConnell, J. D. C. J. Chem. Phys. 1991, 94, 7434-7445.

(25) Smith, D. Langmuir 1998, 14, 5959Ü5967.

(26) Berendsen, H. J. C.; Grigera, J. R.; Straatsma, T. P. J. Phys. Chem. 1987, 14, 6269-6271.

(27) Frenkel, D.; Smit, B. Understanding molecular simulation: from algorithms to applications, 2d edition; Academic Press: London, 2002.

(28) Hensen, E.; Tambach, T.; Bliek, A.; Smit, B. J. Chem. Phys. 2001, 115, 3322-3329.

(29) Botan, A.; Rotenberg, B.; Marry, V.; Turq, P.; Noetinger, B. J. Phys. Chem. C 2010, 114, $14962-14969$. 
(30) Lide, D. R. CRC Handbook of Chemistry and Physics 2010; CRC Press, 2010.

(31) Plimpton, S. J. Comp. Phys. 1995, 117, 1-19, http://lammps.sandia.gov.

(32) Ryckaert, J.-P.; Ciccotti, G.; Berendsen, H. J. Comput. Phys 1977, 23, 327-341.

(33) Liu, P.; Harder, E.; Berne, B. J. J. Phys. Chem. B 2004, 108, 6595-6602.

(34) Rotenberg, B.; Marry, V.; Malikova, N.; Turq, P. Journal of Physics: Condensed Matter 2010, $22,284114$.

(35) Todd, B. D.; Evans, D. J.; Daivis, P. Phys. Rev. E 1995, 52, 1627-1638.

(36) Předota, M.; Cummings, P. T.; Wesolowski, D. J. J. Phys. Chem. C 2007, 111, 3071-307910.

(37) Martí, J.; Sala, J.; Guàrdia, E.; ; Gordillo, M. C. Phys. Rev. E 2009, 79, 031606.

(38) Yeh, I.-C.; Hummer, G. J. Phys. Chem. B 2004, 108, 15873-15879.

(39) Kerisit, S.; Liu, C. Geochim. Cosmochim. Acta 2010, 74, 4937 - 4952.

(40) Rotenberg, B.; Marry, V.; Vuilleumier, R.; Malikova, N.; Simon, C.; Turq, P. Geochim. Cosmochim. Acta 2007, 71, 5089 - 5101.

(41) Ting Chen, B. S.; Bell, A. T. J. Chem. Phys. 2009, 131, 246101.

(42) Melchionna, S.; Marini Bettolo Marconi, U. Europhys. Lett. 2008, 81, 34001.

(43) Bocquet, L.; Barrat, J.-L. Phys. Rev. E 1994, 49, 3079-3092.

(44) Marry, V.; Dufrêche, J. F.; Jardat, M.; Turq, P. Mol. Phys. 2003, 101, 3111-3119.

(45) Lopez-Duran, J.; Khaldoun, A.; Kerkeb, M.; Ramos-Tejada, M.; Gonzalez-Caballero, F. Clays and Clay Min. 2003, 51, 65-74. 
Table 1: Relative error on the overall flux, compared to molecular dynamics simulation, assuming a parabolic profile with stick or slip boundary conditions, with the same fluid viscosity (see also Figure 7).

\begin{tabular}{ccc}
\hline$H(\mathrm{~nm})$ & $\left|J_{\text {stick }}-J_{\mathrm{MD}}\right| / J_{\mathrm{MD}}$ & $\left|J_{\text {slip }}-J_{\mathrm{MD}}\right| / J_{\mathrm{MD}}$ \\
\hline \hline 4.5 & $27 \%$ & $2.7 \%$ \\
8.0 & $15 \%$ & $0.8 \%$ \\
\hline
\end{tabular}


Figure 1: Snapshot of the simulation box. Green: $\mathrm{Al}$ and $\mathrm{Mg}$; yellow: $\mathrm{Si}$; red: O; white: H; blue: $\mathrm{Na}$. Bright atoms illustrate the simulation box for 3D periodic boundary conditions, both bright and shaded atoms - 2D periodic boundary conditions.

Figure 2: (a) Mass density profiles for a pore size $H=4.5 \mathrm{~nm}$. (b) Number density progiles for $\mathrm{Na}, \mathrm{O}$ and $\mathrm{H}$ atoms. $\rho_{\mathrm{Na}}$ was multiplied by a factor of 10 for better visualization. The extreme $z$ values correspond to the location of surface oxygen atoms.

Figure 3: Two-dimensional radial distribution functions for O-atoms calculated, in selected layers of the $10 \mathrm{~nm}$ pore. Each layer is a slab parallel to the clay surface. The gray stripes on the inset indicate the location of the layers with respect to the mass density profile. Results in the d) and all subsequent layers (not shown) almost coincide with the bulk one, shown for comparison.

Figure 4: Water diffusion coefficient parallel $D_{\|}$and perpendicular $D_{\perp}$ to the surface as a function of the distance to the surface, for the largest pore $(\mathrm{H}=10 \mathrm{~nm})$. The stripes indicate the location of the layers in which the diffusion coefficient are calculated.

Figure 5: Steady-state velocity profile in the presence of a force parallel to the surface applied to each fluid atom. Note that the same force is applied in all cases. Symbols indicate the values obtained from NEMD simulations, solid lines are parabolic fits. The vertical dashed lines indicate the location of surface oxygen atoms.

Figure 6: (a) Velocity and density profiles, (b) shear rate and shear stress, (c) viscosity and density profiles, in the $4.5 \mathrm{~nm}$ pore.

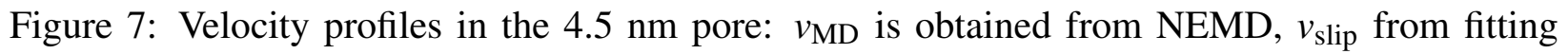
$v_{\text {MD }}$ to a parabolic profile and $v_{\text {stick }}$ by assuming the same viscosity and a vanishing velocity at the Gibbs dividing surface. The slip length computed from Eq. (3) is visible from the red short-dotted linear extrapolation of $v_{\text {slip }}$ beyond the Gibbs dividing surface (vertical solid line). The vertical dashed line indicates the location of surface oxygen atoms. 


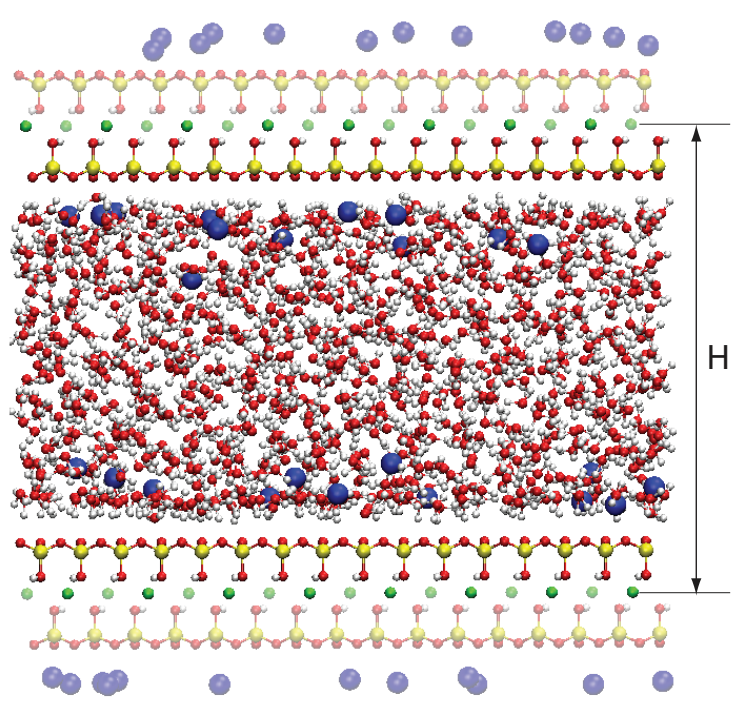

Figure 1.

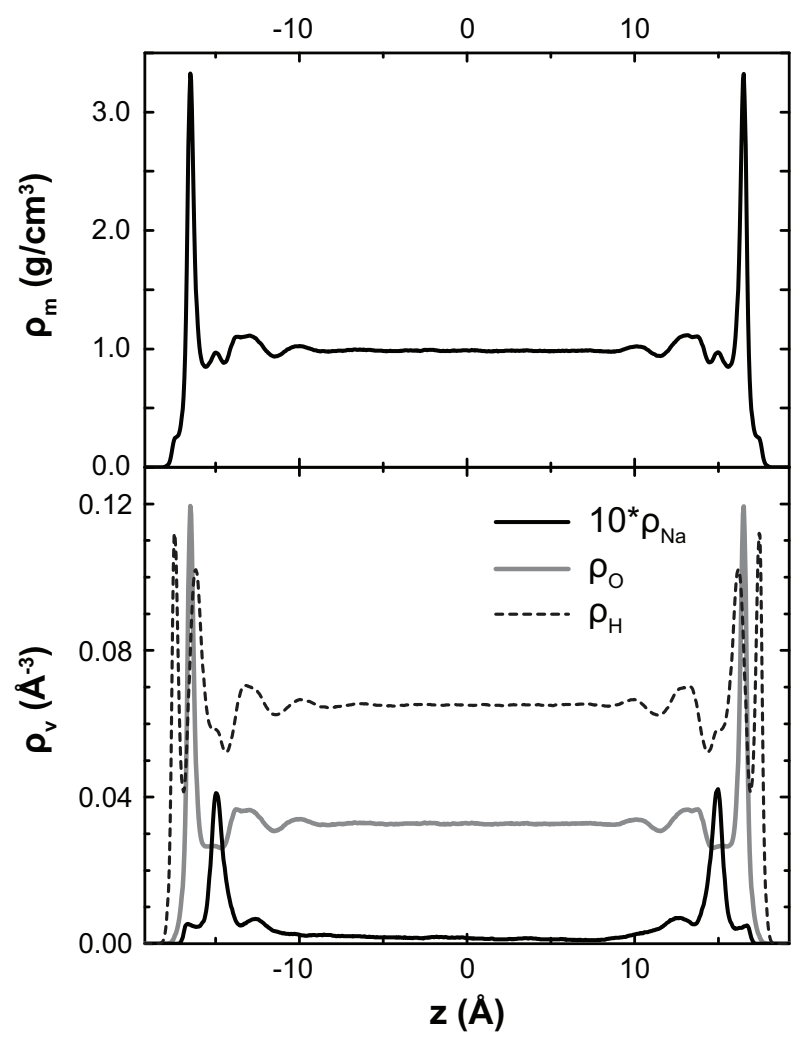

Figure 2. 


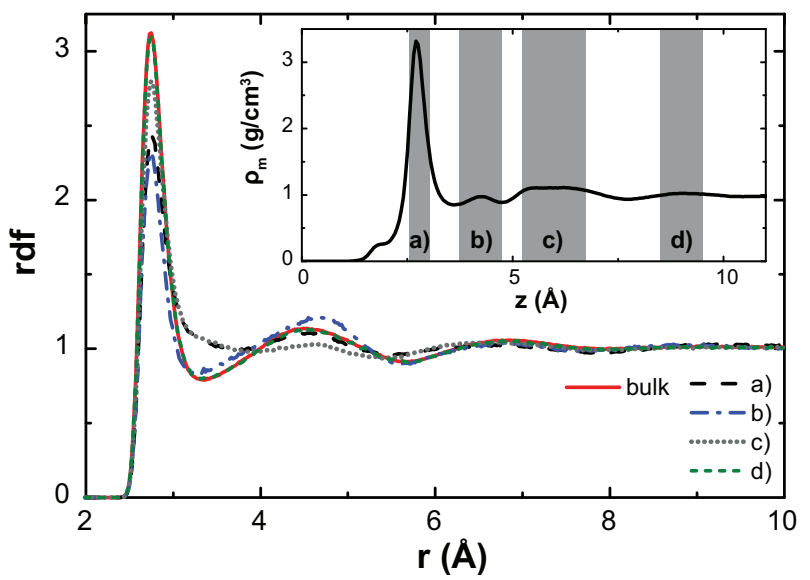

Figure 3.

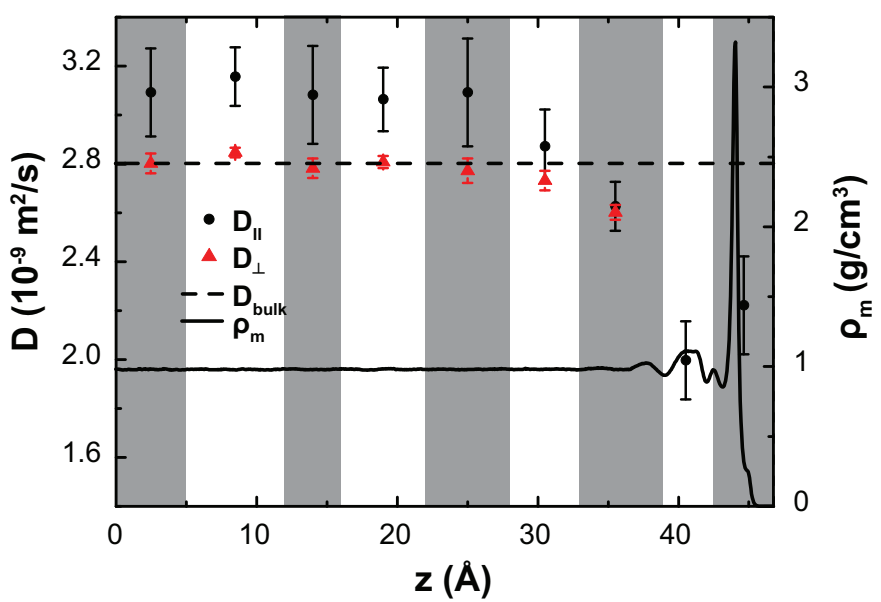

Figure 4.

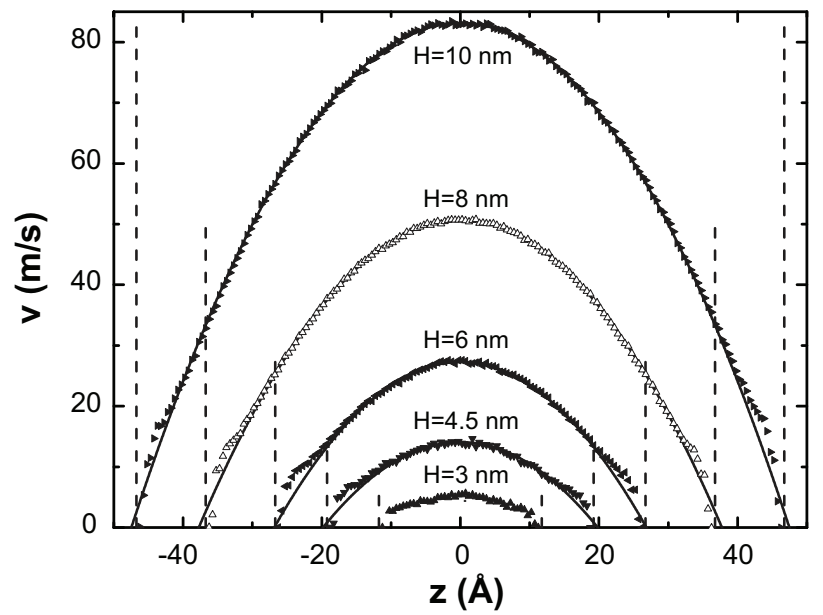

Figure 5. 


\section{Page 21 of 21}

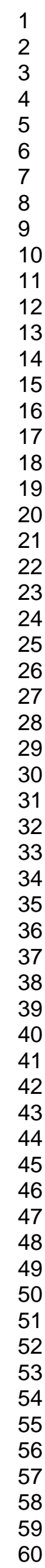
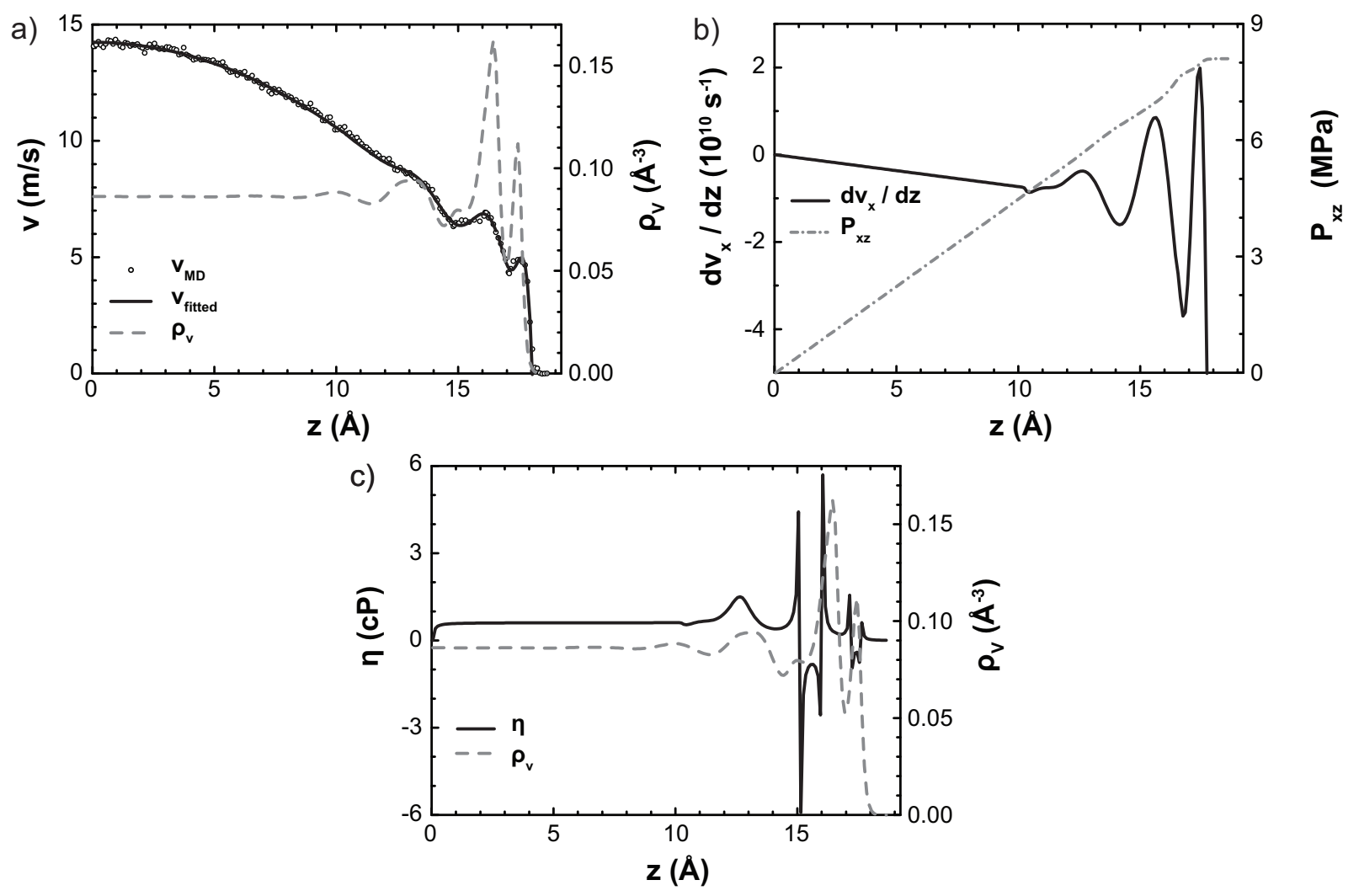

Figure 6.

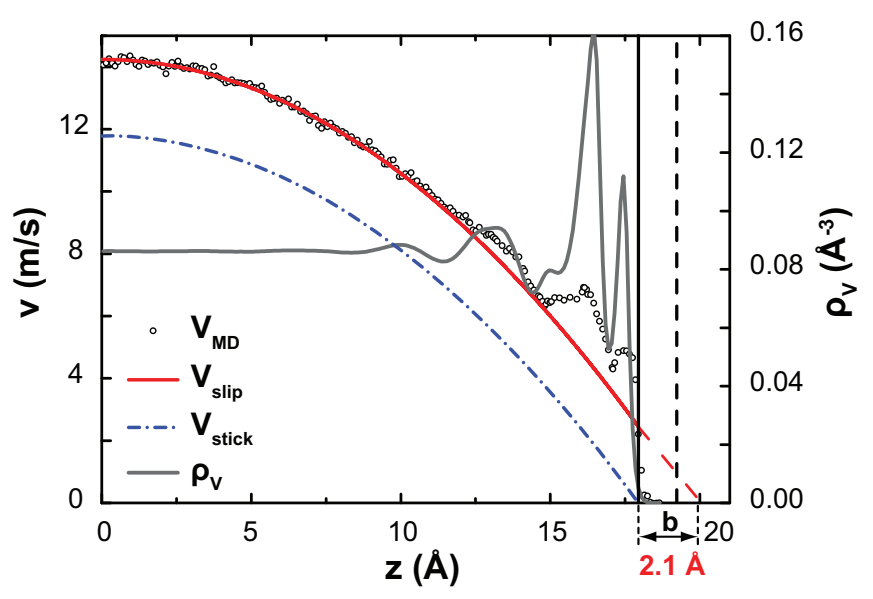

Figure 7. 